\title{
Mobility-aware design of cognitive radio networks: challenges and opportunities
}

\author{
Luca De Nardis \\ INFO-COM Department \\ Sapienza University of Rome \\ Rome, Italy \\ Email: lucadn@newyork.ing.uniroma1.it
}

\author{
Maria-Dolores Perez Guirao \\ Leibniz University of Hannover \\ Hannover, Germany \\ Email: dolores.perez@ikt.uni-hannover.de
}

\begin{abstract}
This work deals with mobility-related issues in cognitive radio network design. With few notable exceptions, research activity on cognitive radio networks focused until now on the case of static networks, neglecting to take into account the impact of mobility on network design. In this work the key issues posed by mobility at the different layers of the protocol stack are discussed, and a case study focusing on routing in cognitive Ultra Wide Band networks is presented, in order to quantitatively assess the impact of mobility on network performance. Next, existing solutions for mobility management in cognitive networks are reviewed, and open issues and future research lines for mobiler cognitive network design are identified. The analysis leads to the conclusion that mobility-related issues should be addressed from a network-wide point of view, taking advantage of terminal cooperation at the local and global level in order to effectively manage mobility in cognitive networks.
\end{abstract}

\section{INTRODUCTION}

Cognitive radios are conceived to operate across different spectrum bands and heterogeneous radio access technologies, driven by the exciting vision of an "always best connected" society that will enable innovative ways of social interaction and personalized services and applications. In this context, the absence of, or even the introduction of significant limitations on, user mobility is unsustainable.

Node mobility significantly affects the performance of wireless networks and it is a common cause of route outages, packet loss and delay. In traditional, centralized wireless networks, mobility management traditionally involves two tasks: location and handover management. Both tasks rely on the logical division of the network coverage area into administrative domains, such as clusters or cells. Location management tracks the location of the network nodes, while handover management is responsible for keeping end-to-end connections active while involved nodes move from one administrative domain to another [1]. In most application scenarios, however, such an approach cannot be extended in a straightforward way to cognitive radio networks due to the lack of a centralized network architecture, and specific solutions for mobility management are required.

The state of the art research on cognitive radio networks has focused on the time varying character of the available spectrum resources, leaving specific terminal mobility issues mostly unexplored. Most spectrum sensing [2], [3] and dynamic spectrum access [4] approaches, thus far, address cognitive radio networks with static topology. This limitation is just not sustainable in the light of the economic and social value that mobile cognitive radio networks can bring. On the other hand, the consideration of node mobility imposes new challenges, making it necessary to review current network and protocol design approaches for, among others, spectrum sensing, spectrum sharing, interference management and routing.

The analysis of mobility-related issues in cognitive radio networks and the design of mobility-aware solutions are indeed the main focus of the Special Interest Group 3 created within the EU-funded COST Action IC0902 [5], launched in December 2009 with the aim of coordinating, supporting and fostering research activities focusing on all key aspects in the design of cognitive radio and networks both within Europe and world-wide.

In this framework, this work addresses the specific issues related to mobility in the design of a network of mobile cognitive terminals. First, the impact of mobility on different layers of the protocol stack is analyzed and exemplified by means of a specific case study. Next, existing solutions for coping with mobility in cognitive radio networks are discussed. Finally, open issues and future research lines related to mobility in heterogeneous cognitive radios are discussed.

The paper is organized as follows. Section II discusses the problems posed by mobility in cognitive networks at physical, link and network layers. Next, Section III provides a quantitative analysis of the impact of mobility in a specific case study, dealing with routing in a UWB cognitive radio. Section IV reviews existing approaches to mobility management in cognitive radios. Finally, Section V discusses open issues and potential solutions for the introduction of mobility-related mechanisms in the design of cognitive networks.

\section{MOBILITY IN COGNITIVE RADIO NETWORKS DESIGN}

Mobility management in conventional cellular and mobile ad hoc networks (MANETs) is in charge of facilitating reliable end-to-end connectivity and QoS provisioning under timeand location-varying operation conditions. In cognitive radio networks, this task becomes harder, as it has to cope not only with changes in the terminals' physical location but possibly changes in the terminals' operation frequency due to primary 
user (PU) activity, with incidental adjustment of the wireless access technology and extensive protocol reconfiguration. As a necessary step to understand the implications of mobility in cognitive radio networks, the following subsections introduce the specific challenges and opportunities imposed by terminal and spectrum mobility at the different layers of the protocol stack in cognitive radio networks.

\section{A. Physical layer}

Spectrum sensing is instrumental to context-awareness at the physical layer. It enables cognitive radio networks to opportunistically exploit unused spectrum resources while preventing harmful interference to PUs. Sensing control, involving dynamic scheduling of sensing periods (sensing scheduling) [2] and fusion of distributed sensing information (cooperative sensing) [6], [7], [8], is considered an efficient way to improve sensing accuracy by exploiting spatio-temporal diversity in the captured signals. Under appropriate sensing control, terminal mobility can significantly improve sensing performance by augmenting the spatial diversity of the sensed samples. The work in [9] has analytically confirmed this intuition, and proven that the speed of the mobile sensors directly impacts the contribution of sensing scheduling to the overall sensing performance gain.

\section{B. Link layer}

A general assumption about cognitive radio networks is their heterogeneous nature; that is, it is assumed that cognitive radio terminals will be able to opportunistically operate over several spectrum bands and employ multiple radio access technologies (RATs). In order to establish communication, cognitive radio terminals need to know which other terminals operate with the same RAT and in the same spectrum band. In a mobile network, cognitive radio terminals must thus periodically perform neighbour discovery, also referred to as link rendezvous [10], on a common control channel in order to discover new neighbours and obtain information about their capabilities and network parameters, as well as exchange position and topology information. However, establishing common control channels is made difficult by the dynamic availability of spectrum resource derived from PU activity and the additional variability introduced by the mobility of terminals. The impact of mobility can be somehow reduced by taking it into account in the design of MAC and network organization protocols; as an example, an estimation of terminal mobility could be used in measuring the suitability of a terminal to play a role in the local network organization.

A second key aspect in the design of link layer mechanisms that must take into account mobility is related to spectrum mobility or spectrum handover procedures [4], to be carried out when a cognitive radio has to abandon the current channel due to PU activity and resume transmission in another vacant channel, possibly without disrupting end-to-end connectivity. Mobility of candidate terminals should be taken into account when selecting the new channel configuration, in order to minimize the number of handovers and the corresponding perturbations to end-to-end connectivity.

\section{Network layer}

Despite the possible solutions to optimize link layer protocols for cognitive networks, link layer solutions are only effective when a limited portion of the network is affected by the activity of PUs. When the activity of PUs impacts several links an efficient reaction requires a joint action at network and link layers. Hence, at the network layer routing protocols have to be coupled with radio resource management and spectrum handover functions. For optimal route computation, the routing tables have to be extended to include context specific information such as the frequency and propagation characteristics of the operation channel, link quality indicators, end-to-end performance requirements, expected PU activity or predicted node mobility. At the same time, simple distance minimisation techniques cannot be longer used and novel multi-dimensional optimisation metrics must be designed. Most of the existing routing approaches achieve this by means of novel context-aware routing metrics [11], [12]. In [11] the authors propose a routing metric that models the endto-end delay by taking into account the number of hops, the number of spectrum handovers along the path and the frequency of spectrum handovers over each link in the path. The computation of this routing metric is possible at each node in the network because it is assumed that each node knows, thanks to an information exchange protocol, the global network topology and the channels available to each other node. The proposed information exchange protocol, based on a broadcast packet exchange, is however only tested in a very favorable scenario, characterized by an error-free channel and collision-free medium access. In [12] the authors propose a probabilistic routing approach based on the assumption that spectrum opportunities and thus link connectivity in cognitive radio networks are not likely to be random but rather follow a learnable pattern.

\section{IMPACT OF MOBILITY ON COGNITIVE RADIO NETWORKS: A CASE STUDY}

This section presents an example of the impact of mobility on the performance of cognitive radio networks, focusing on the routing layer. A routing strategy capable of taking into account the impact of primary transmitters on the performance of a secondary network of UWB devices was originally proposed in [13], where a link cost function obtained as a linear combination of terms taking into account different aspects of the UWB terminals was defined. The cost of a link between two generic terminals $x$ and $y$ is defined as follows:

$$
\begin{aligned}
& U W B_{\text {Cost }}(x, y)= \\
& c_{\text {Sync }} \cdot \operatorname{Sync}(x, y)+c_{\text {Power }} \cdot \text { Power }(x, y)+ \\
& +c_{M U I} \cdot \operatorname{MUI}(x, y)+c_{\text {Reliability }} \cdot \text { Reliability }(x, y)+ \\
& +c_{\text {Traffic }} \cdot \text { Traffic }(y)+c_{\text {Delay }} \cdot \text { Delay }(x, y)+ \\
& +c_{\text {Aut }} \cdot \text { Autonomy }(y)+c_{\text {Coex }} \cdot \text { Coexistence }(y)
\end{aligned}
$$

The coexistence term relies on the capability of UWB devices to efficiently detect the presence of primary transmitters, 
TABLE I

SiMULATION SETTINGS

\begin{tabular}{|c||c|}
\hline \multicolumn{1}{|c||}{ Parameter } & Value(s) \\
\hline Area & $400 \mathrm{x} 400 \mathrm{~m}^{2}$ \\
\hline Number of terminals $N_{U W B}$ & 50 \\
\hline Network topology & Random node positions \\
\hline Number of runs & 10 \\
\hline Simulation time & $10000 \mathrm{~s}$ \\
\hline Transmission rate & $966 \mathrm{~kb} / \mathrm{s}$ \\
\hline Transmission power & $36.5 \mu W$ \\
\hline Physical layer settings & Impulse Radio, Bandwidth $=500$ \\
& MHz \\
\hline Access strategy & Pure Aloha \\
\hline Traffic model & CBR @ 20 kb/s \\
\hline Average conn. duration & $12.5 \mathrm{~s}$ \\
\hline Average conn. request interval & $250 \mathrm{~s}$ \\
\hline Channel model & IEEE $802.15 .4 \mathrm{a}$ outdoor LOS $[14]$ \\
\hline Mobility model & Gauss-Markov [15] \\
\hline
\end{tabular}

by assigning a higher cost to links involving terminals that sense a strong interference as follows:

$$
\text { Coexistence }(y)=\frac{\text { MeasuredExternalInterference }(y)}{\text { MaximumInterference }(y)} \text {. }
$$

Details on the other terms are out of the scope of this work, and can be found in [13].

The performance of the proposed strategy was analyzed in a static network assuming that a central entity performed an optimal route selection based on the knowledge of costs of all network links. The proposed strategy led to higher throughput and lower energy consumption thanks to a lower number of packet errors caused by external interference [13].

In this work the strategy was revisited by taking into account the impact of terminal mobility on network performance. The scenario considered in the analysis consisted in a network of $N_{U W B}$ UWB mobile terminals coexisting with a set of $N_{P r i}$ primary transmitters. A source UWB terminal generated traffic connection request towards a destination positioned on the opposite side of the simulation area relying on multi-hop routes through intermediate terminals for delivering packets. Following a connection request, a Dynamic Source Routing protocol was used for path search, combined with either of two different strategies: a) a strategy adopting a traditional metric minimizing the number of hops and b) a strategy adopting the metric defined in eq. (1), referred in the following as Hops and Cost strategies, respectively. For the Cost strategy the following coefficient set was adopted: $c_{S y n c}=c_{M U I}=$ $c_{\text {Reliability }}=c_{\text {Traffic }}=c_{\text {Aut }}=0, c_{\text {Power }}=10^{-9}, c_{\text {Delay }}=$ $10^{-10}, c_{\text {Coex }}=1$. Parameters related to the simulation scenario and to the UWB terminals are presented in Table I.

Both source and destination terminals were kept in fixed positions in all simulation runs. In a first simulation scenario the intermediate terminals were kept still as well, and the network performance was analyzed as a function of the
TABLE II

CHARACTERISTICS OF THE PRIMARY TRANSMITTERS

\begin{tabular}{|c|c|}
\hline Parameter & Value(s) \\
\hline Number $N_{P r i}$ & from 0 to 3 \\
\hline Position & Randomly selected \\
\hline Duty cycle during an activity period & 0.001 \\
\hline Average duration of each sleep/activity period & $1 \mathrm{~s} / 10 \mathrm{~s}$ \\
\hline Carrier frequency & $3.5 \mathrm{GHz}$ \\
\hline Transmission bandwidth & $40 \mathrm{MHz}$ \\
\hline Transmit power $P_{\text {Int }}$ & $1 \mathrm{~W}, 10 \mathrm{~W}$ \\
\hline
\end{tabular}

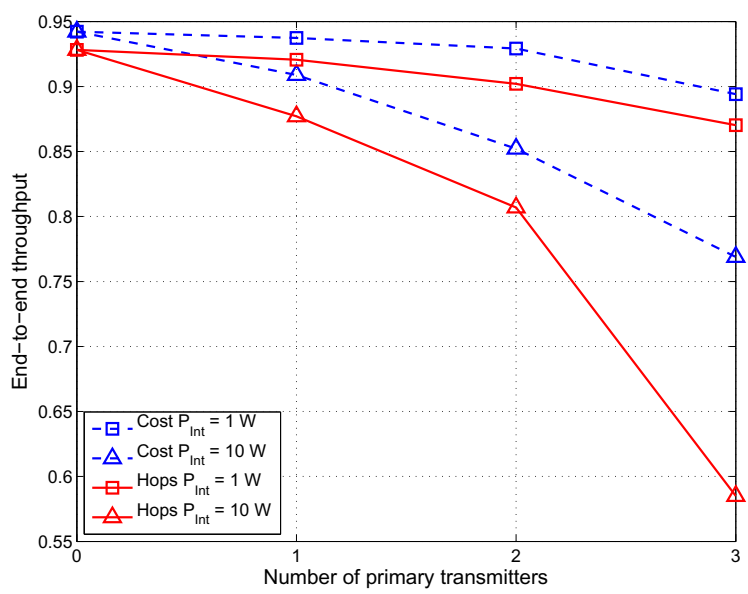

Fig. 1. End-to-end throughput for the Hops vs. Cost routing strategies as a function of the number of Primary Transmitters for different transmit powers.

number of primary transmitters in the area and of their transmit power, as detailed in Table II, reporting the key parameters related to the primary transmitters. Figure 1 presents the endto-end throughput for this scenario, comparing the Hops vs. Cost routing strategies, and shows that the introduction of information related to the radio environment in the routing function can dramatically improve the network performance even in a more realistic simulation scenario than the one adopted in [13]. The improvement is achieved by adopting routes that avoid network regions affected by the presence of nearby primary transmitters, as shown in Figure 2, presenting the average number of hops per connection in the same simulation scenario. Figure 2 highlights that the Cost routing strategy leads to a higher average number of hops per connection, in order to avoid interference-prone terminals.

On the other hand, one can reasonably expect a strategy selecting longer routes to be significantly affected by mobility, due to a higher probability of route disconnection when compared to a hops-minimizing strategy. In order to investigate this aspect, in a second set of simulations part of the intermediate terminals were allowed to move according to the Gauss-Markov [15] mobility model with a maximum speed of $1 \mathrm{~m} / \mathrm{s}$. Three different mobility scenarios were considered, characterized by a percentage of the intermediate terminals allowed to move equal to $25 \%, 50 \%$ and $75 \%$ respectively. 


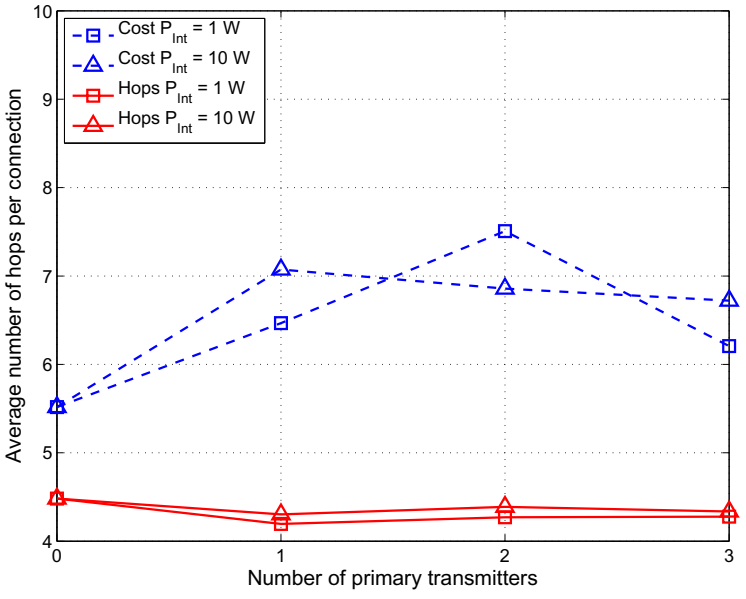

Fig. 2. Average number of hops per connection for the Hops vs. Cost routing strategies as a function of the number of Primary Transmitters for different transmit powers.

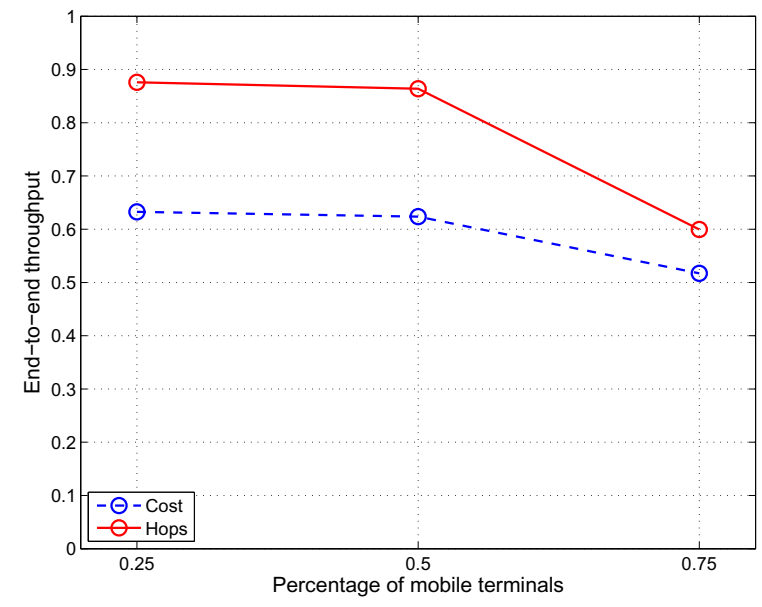

Fig. 3. End-to-end throughput for the Hops vs. Cost routing strategies as a function of the percentage of intermediate terminals allowed to move.

Results on end-to-end throughput in the second scenario are presented in Figure 3.

Results highlight that the cognitive routing strategy leads to worse network performance when compared to a straightforward minimization of the number of hops, even when full information on the presence of primary transmitters is available at each secondary network terminal, and suggest thus that mobility should be explicitly taken into account in the design of a cognitive radio network. In this view, Section IV reviews some mobility management solutions taking into account the specifics of cognitive radio networks.

\section{SOLUTIONS FOR MOBILITY MANAGEMENT IN COGNITIVE RADIO NETWORKS}

Specific solutions for mobile cognitive radio networks are still scarce in the literature. Most, if not all, solutions target the network and transport layer and are therefore independent of the underlying RAT. Key aspects of such solutions are presented in the following subsections.

\section{A. Network Layer}

The geographic forwarding based spectrum aware routing protocol (SEARCH) [16] supports joint route and spectrum selection in order to minimise the end-to-end path latency due to spectrum handover along the route. For this, the protocol tries to avoid regions of high PU activity during route formation, while taking into account node mobility concerns. Consider that nodes forming a connected route free of PU activity may move into the coverage area of a PU on the current channel, thus detaining transmission and forcing the nodes to either bypass the region by changing the route or to switch the current channel immediately. The key novelty of SEARCH is that its route formation procedure does not associate routes with particular nodes, but with anchor points that represent regions free of PU activity. Through periodic beaconing on a control channel, nodes are kept aware of the current locations of their one-hop neighbours. When a forwarding node detects that the distance of the next hop in the route from the relevant anchor point is larger than a predefined threshold, it initiates a route reconfiguration process with the goal to find a new next hop closest to the anchor point. Since the source and destination nodes cannot be replaced in the route, their mobility has to be tackled in a different way. Destination (source) nodes predict their future locations through Kalman filtering and inform their previous (next) hop node. If the previous (next) hope detects that the destination (source) node has become unreachable, it initiates a new route formation procedure that may add new nodes to the existing path. For highly mobile environments, this approach may result in long, sub-optimal paths.

\section{B. Transport Layer}

Pioneering work at the transport layer has been presented in [17], where the authors propose a window-based protocol addressing specific challenges of cognitive radio networks. The protocol is capable of distinguishing between different factors that affect end-to-end flow performance and conduct different recovery mechanisms accordingly. Precisely, the protocol is capable of distinguishing cognitive radio specific issues such as link interruptions due to spectrum sensing and spectrum mobility from classical network congestion issues and link failures due to node mobility. This capability relies on enhanced context-awareness at the source achieved by explicit feedbacks from intermediate nodes and the destination. The weak point of this approach is that the same factors that the source would like to differentiate also affect the packet delivery performance on the feedback path. Thus, the feedback path may be unreliable and induce severe delay and packet losses to the control messages. In order to make this approach more robust, two directions may be adopted: (i) to explore techniques to increase the packet delivery reliability on the feedback path, for instance, the use of out-of-band control channels or (ii) explore techniques to reduce the dependence of the protocol on explicit feedback by, for instance, improving the predictive capabilities of the source. 


\section{CONCLUSION AND DISCUSSION}

This work addressed opportunities and open issues related to the introduction of mobility in cognitive wireless networks. The impact of mobility on physical layer, link layer and network layer design in cognitive networks was analyzed, and a case study focusing on routing in a cognitive UWB network was presented, in order to quantitatively assess the potential impact of mobility on network performance. Results highlighted that a solution appositely designed to improve performance in a cognitive secondary network can actually lead to poor results if terminal mobility is not taken into account in the design. In this view, existing approaches for mobility management in cognitive networks were reviewed. Research on mobility management has been until now mainly focusing on mobility prediction schemes that assume the position information to be available at each network terminal at all times by means of either dedicated hardware (GPS) or a reliable location server. Mobility prediction is thus typically demanded to each single network terminal, which is in charge of determining its own mobility pattern and react to it.

Such an approach neglects however two key aspects that should be taken into account in the design of mobility-aware solutions for cognitive networks. First, position information can be incomplete or missing, for example if a distributed positioning algorithm is used to obtain position information; second, mobility patterns of different network terminals can be heavily correlated, depending on the considered application scenario, and mobility management schemes that work independenly on each terminal cannot take advantage of such correlation.

Both aspects suggest that mobility can be handled more efficiently by taking advantage of cooperation between network terminals: as an example, terminals cooperating in the retrieval of position information by means of distributed positioning can contextually determine and track their mobility patterns by mutual information exchange. In the context of the case study presented in this work this could lead to the introduction of a mobility-aware cost term that takes into account the mobility of each terminal related to the mobiliy of its neighbours. In a similar fashion, terminal cooperation could enable specific solutions to be adopted for all the open issues identified in Section II. More in general, the analysis carried out in this work suggests that mobility should be treated as a network-wide issue, taking advantage of cooperation in order to contribute to the creation of a full context-awareness, that is the capability to capture and fully understand the network operation environment. The process of acquiring contextawareness can be compared to the process of solving a puzzle for which the pieces (mostly in form of meters giving an indication of some quantity) are distributed across the layers of the protocol stack of the cognitive radio terminals, and in this framework the information related to mobility of single terminals as well as of groups of terminals can play a key role in the design of efficient solutions for mobile cognitive networks.

\section{ACKNOWLEDGMENT}

This work was supported in part by COST Action IC0902 "Cognitive Radio and Networking for Cooperative Coexistence of Heterogeneous Wireless Networks".

\section{REFERENCES}

[1] I. Akyildiz, J. Xie, and S. Mohanty, "A survey of mobility management in next-generation all-ip-based wireless systems," Wireless Communications, IEEE, vol. 11, no. 4, pp. 16-28, Aug. 2004.

[2] Y.-C. Liang, Y. Zeng, E. Peh, and A. T. Hoang, "Sensing-throughput tradeoff for cognitive radio networks," Wireless Communications, IEEE Transactions on, vol. 7, no. 4, pp. 1326 -1337, april 2008.

[3] W.-Y. Lee and I. Akyildiz, "Optimal spectrum sensing framework for cognitive radio networks," Wireless Communications, IEEE Transactions on, vol. 7 , no. 10 , pp. $3845-3857$, october 2008 .

[4] I. F. Akyildiz, W.-Y. Lee, M. C. Vuran, and S. Mohanty, "Next generation/dynamic spectrum access/cognitive radio wireless networks: a survey," Comput. Netw., vol. 50, no. 13, pp. 2127-2159, 2006.

[5] http://w3.cost.esf.org/index.php?id=110action_number=IC0902, "Cost action ic0902 website."

[6] S. Mishra, A. Sahai, and R. Brodersen, "Cooperative sensing among cognitive radios," vol. 4, june 2006, pp. $1658-1663$.

[7] Z. Quan, S. Cui, H. Poor, and A. Sayed, "Collaborative wideband sensing for cognitive radios," Signal Processing Magazine, IEEE, vol. 25, no. 6, pp. $60-73$, november 2008.

[8] L. De Nardis and M.-G. Di Benedetto, "Clustered Hybrid Energy-aware cooperative Spectrum Sensing (CHESS)," in IEEE Cognitive Radio Oriented Wireless Networks and Communications 2009 (CROWNCOM 2009), June 2009, pp. 1-6.

[9] A. W. Min and K. G. Shin, "Impact of mobility on spectrum sensing in cognitive radio networks," in CoRoNet '09: Proceedings of the 2009 ACM workshop on Cognitive radio networks. New York, NY, USA: ACM, 2009, pp. 13-18.

[10] L. DaSilva, Rendezvous in cognitive radio networks., n. E. In: Fette B. Cognitive Radio Technology, Ed. Elsevier, Amsterdam, 2009.

[11] S. Krishnamurthy, S. Venkatesan, and R. Prakash, "Control channel based mac-layer configuration, routing and situation awareness for cognitive radio networks," in in Proceedings of MILCOM 2005, 2005.

[12] A. C. Talay and D. T. Altilar, "Racon: a routing protocol for mobile cognitive radio networks," in CoRoNet '09: Proceedings of the 2009 $A C M$ workshop on Cognitive radio networks. New York, NY, USA: ACM, 2009, pp. 73-78.

[13] M.-G. Di Benedetto and L. De Nardis, "Cognitive routing models in UWB networks," in IEEE International Conference on Cognitive Radio Oriented Wireless Networks and Communications (CROWNCOM), May 2008, pp. 1-6.

[14] "IEEE 802.15.4a channel model final report, rev.1 (november 2004)," Available at: ftp://ieee:wireless@ftp.802wirelessworld.com/15/ 04/15-04-0662-00-004a-channel-model-final-report-r1.pdf, November 2004.

[15] B. Liang and Z. J. Haas, "Predictive distance-based mobility management for multidimensional PCS networks," IEEE/ACM Transactions on Networking, vol. 11, no. 5, pp. 718-732, June 2003.

[16] K. Chowdhury and M. Felice, "SEARCH: A Routing Protocol for Mobile Cognitive Radio Ad-Hoc Networks," Computer Communications, vol. 32, no. 18, pp. 1983 1997, 2009, cognitive Radio and Dynamic Spectrum Sharing Systems. [Online]. Available: http://www.sciencedirect.com/science/ article/B6TYP-4WMM7DS-1/2/e2ba46e19d1d0c8189a178202085e8af

[17] K. Chowdhury, M. Di Felice, and I. Akyildiz, "Tp-crahn: a transport protocol for cognitive radio ad-hoc networks," in INFOCOM 2009, IEEE, April 2009, pp. 2482-2490. 